\title{
Silicon-Photonics Devices for Low-Power, High-Bandwidth Optical I/O
}

\author{
J. Van Campenhout, M. Pantouvaki, P. Verheyen, H. Yu ${ }^{1}$, P. De Heyn ${ }^{1}$, G. Lepage, W. Bogaerts ${ }^{1}$, P. Absil \\ imec, Kapeldreef 75, 3001, Leuven, Belgium \\ ${ }^{I}$ Photonics Research Group, Dept. of Information Technology, Ghent University - imec, Center of Nano- and Biophotonics (NB Photonics), \\ St. Pietersnieuwstraat 41, 9000 Ghent, Belgium \\ Author e-mail address:jvcampen@imec.be
}

\begin{abstract}
Electro-optic transceivers integrated in silicon-photonics interposers are attractive for realizing low-power high-bandwidth Optical I/O for future advanced logic and memory. We review recent results obtained at imec on low-voltage silicon ring modulators and $\mathrm{Ge}$ photodetectors.
\end{abstract}

OCIS codes: (200.4650) Optical interconnects; (250.4110) Modulator; (250.0040) Detectors

\section{Silicon-Photonics Interposer Technology for 3-D Optical I/O}

Aggregate bandwidth requirements for I/O in advanced CMOS chips and stacked DRAM packages are expected to reach the level of multiple TB/s by 2018. Given the constrained I/O power envelope, the energy efficiency for a proposed silicon-based Optical I/O solution will have to be on the order of $1 \mathrm{pJ} / \mathrm{bit}$ or better [1]. In addition, I/O bandwidth densities from the chip edge into optical fiber (ribbons) on the order of $1 \mathrm{~Tb} / \mathrm{s} / \mathrm{mm}$ will have to be obtained, likely requiring the introduction of wavelength-division multiplexing (WDM) techniques.

Obtaining such power efficiencies and bandwidth densities within the voltage constraints of advanced CMOS circuits will require the adoption of the best-in-class silicon optical devices, combining low-loss optical channels with high modulation and photodetection efficiencies at low applied voltages. Although monolithic integration of photonics into the CMOS flow would arguably enable the highest integration density and best power efficiencies, co-integration of high-performance silicon optical devices becomes increasingly more challenging in advanced CMOS nodes, and it is not clear if sufficient compound yield can be obtained at low cost.

An attractive alternative for silicon-based Optical I/O involves the 3-D flip-chip assembly through microbumping [2] of a silicon-photonics interposer with the CMOS logic die and/or DRAM stack, as shown in Fig. 1. Microbumping enables tight, hybrid integration of know-good dies fabricated with distinct optimized technologies within a single package, with low electrical parasitics. The potential of tight-pitch microbumping for realizing silicon optical transceivers with sub-pJ/bit energy efficiency using has already been demonstrated in [3].

In this paper, we review the recent progress at imec on low-voltage, low-loss silicon ring modulators and Ge waveguide photodetectors and their co-integration on a silicon-on-insulator wafer for realizing a high-performance silicon-photonics interposer, as a first step to toward a competitive 3-D Optical I/O technology.

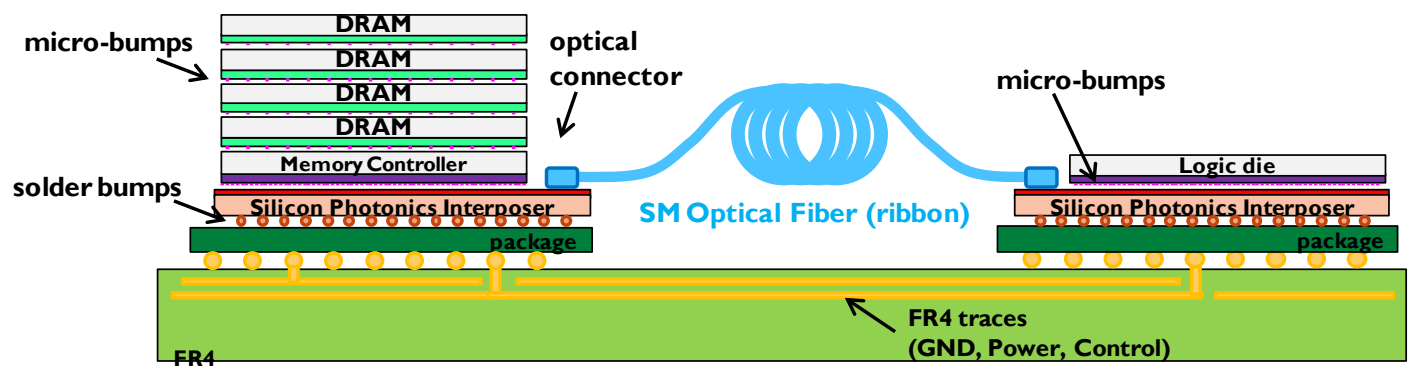

Figure 1: Concept of silicon-photonics interposers for high-bandwidth, low-power 3-D Optical I/O for advanced CMOS logic and DRAM stacks.

\section{Low-loss, low-voltage silicon electro-optic ring modulators}

Silicon ring optical modulators are attracting increasing interest for low energy, small footprint optical interconnects co-integrated with CMOS electronics. Key requirements for such devices are high extinction ratio (ER 10dB), low insertion loss (IL<1dB), high modulation speed ( $>10 \mathrm{~Gb} / \mathrm{s}$ ) and low energy per bit (<50fJ/bit). Recently, $10 \mathrm{~Gb} / \mathrm{s}$ and $25 \mathrm{~Gb} / \mathrm{s}$ optical modulation has been reported in carrier-depletion ring modulators using a $1 \mathrm{~V}$ peak-to-peak driving voltage $\left(\mathrm{V}_{\mathrm{pp}}\right)$ [4]. However, the obtained extinction ratios were limited to $5 \mathrm{~dB}$ for insertion losses lower than $3 \mathrm{~dB}$. Such modulators are typically designed with the depletion area parallel to the waveguide (lateral diode design). Modulators with junctions perpendicular to the waveguide (interdigitated diode design) have been proposed to 
increase the modulation efficiency and extinction ratio, however so far they required high voltage swings $\mathrm{V}_{\mathrm{pp}}$, incompatible with CMOS [5]. We have fabricated and compared the performance of two identical ring modulators, differing only in the diode design [6]. We find that the interdigitated design results in higher modulation efficiency, achieving a $7.5 \mathrm{~dB}$ extinction ratio for $3 \mathrm{~dB}$ insertion loss using $1 \mathrm{~V}_{\mathrm{pp}}$, as opposed to only $3 \mathrm{~dB}$ extinction ratio with the lateral diode design. Open eye diagrams were obtained at $10 \mathrm{~Gb} / \mathrm{s}$ for both modulator types, as illustrated in Fig. $2 \mathrm{c}$ for the interdigitated design. Design improvements are expected to improve the modulation speed of the interdigitated modulator up to $25 \mathrm{~Gb} / \mathrm{s}$. Modulation speeds up to $40 \mathrm{~Gb} / \mathrm{s}$ have already been demonstrated in MachZehnder modulators with traveling-wave electrodes and the conventional lateral diode designs [7].
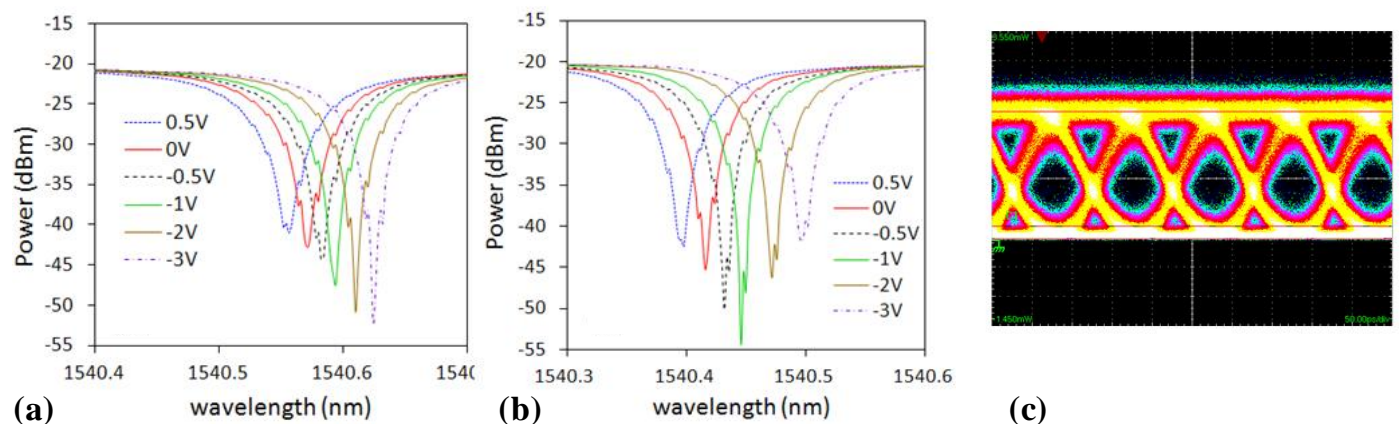

(c)

Figure 2: a) Static through-port transmission spectra showing the resonance wavelength shift vs. applied voltage for depletion ring modulators $(40 \mu \mathrm{m}$ radius) with lateral $(22 \mathrm{pm} / \mathrm{V})$ and b) interdigitated diode design $(30 \mathrm{pm} / \mathrm{V})$. c) $10 \mathrm{~Gb} / \mathrm{s}$ eye diagram at $1.2 \mathrm{~V}_{\mathrm{pp}}$ for a ring modulator with interdigitated diode design [6].

As an alternative to depletion-type modulators, we also explore compact ring modulators based on an embedded metal-oxide-semiconductor (MOS) capacitor (see Fig. 3, [8]). Owing to the high efficiency of the embedded MOSbased phase shifter [6], a MOS ring modulator exhibits a resonance-wavelength shift of more than $130 \mathrm{pm} / \mathrm{V}$, as shown in Fig. 3e. An ER as high as 8dB was obtained together with an IL of only 3dB for a voltage swing of only $1.5 \mathrm{Vpp}$ in a MOS device with a relatively low optical Q of only 3500. Open eye diagrams were obtained up to $3 \mathrm{~Gb} / \mathrm{s}$, limited by the RC cut-off frequency. Proposed design improvements will enable modulation speeds well beyond $10 \mathrm{~Gb} / \mathrm{s}$ in future devices, leveraging the intrinsically fast carrier-accumulation effect. As such, MOS-based ring structures offer a compelling platform for realizing robust, ultra-low voltage silicon modulators, likely surpassing the performance trade-offs that can be obtained with depletion-based ring modulators.
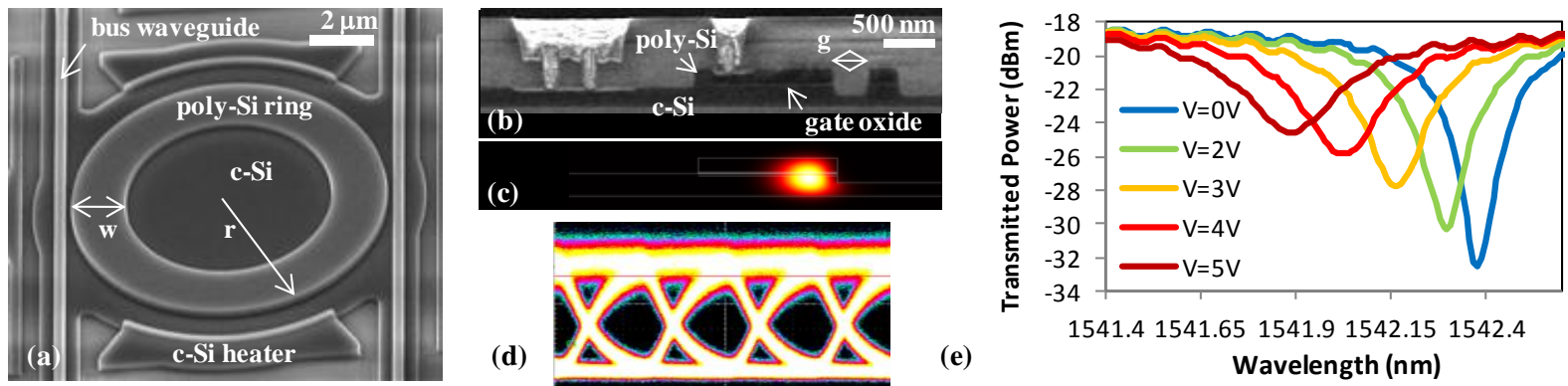

Figure 3: a) Tilted top-view SEM image of a MOS ring modulator, b) cross section SEM image of MOS structure, (c) simulated mode profile of the fundamental whispering-gallery mode, (d) $2 \mathrm{~Gb} / \mathrm{s}$ eye diagram for $1.5 \mathrm{~V}_{\mathrm{pp}}$ drive signal, and (e) static through-port transmission spectra for various applied voltages $(136 \mathrm{pm} / \mathrm{V})[8]$.

\section{High-responsivity Ge-on-Si waveguide photodetectors}

Low-power silicon-based optical transceivers require the co-integration of high-speed Ge waveguide photodetectors with high responsivity and low dark currents on the same substrate as the silicon modulators. We have developed a process module for co-integrating Ge photodetectors with the MOS modulators described in section 2 [10]. The module involves selective Ge epitaxial growth by reduced-pressure chemical-vapor deposition, followed by a postgrowth anneal to remove threading dislocations from the Ge. Subsequently, chemical-mechanical polishing is employed to flatten the Ge patterns. The existing topography in the underlying $\mathrm{Si}$ and poly-Si layers can be used to fabricate "inverted-rib" Ge waveguide structures by deliberate lateral overgrowth of the Ge (see Fig. 4a). Such laterally overgrown areas can be exploited to reduce the optical absorption at metal contacts, by providing lateral confinement away from the lossy metals for the optical mode propagating in the Ge waveguide. 
Lateral p-i-n Ge waveguide photodetectors were fabricated with widths in the range $1.4 \mu \mathrm{m}$ to $3 \mu \mathrm{m}$ and lengths in the range $5 \mu \mathrm{m}$ to $50 \mu \mathrm{m}$. The dark current was measured to be around $2 \mu \mathrm{A}$ at $-1 \mathrm{~V}$ at $25 \mathrm{C}$ (Fig. $4 \mathrm{c}$ ). The photodetector responsivity at $1.5 \mu \mathrm{m}$ wavelength was measured to be around $0.72 \mathrm{~A} / \mathrm{W}$ for a $2 \mu \mathrm{m}$-wide device, and up to $0.9 \mathrm{~A} / \mathrm{W}$ for a $3 \mu \mathrm{m}$-wide device, both $20 \mu \mathrm{m}$ long. For the "inverted-rib" Ge photodetector, the responsivity was as high as $0.9 \mathrm{~A} / \mathrm{W}$ even for a $1 \mu \mathrm{m}$-wide device, owing to the reduced impact from metal absorption losses. Highspeed characterization was also performed for a $5 \times 2 \mu \mathrm{m}^{2} \mathrm{Ge}$ PD. An open eye diagram was obtained at $10 \mathrm{~Gb} / \mathrm{s}$ for 2V (Fig. 4b).

Although the demonstrated levels for dark current and responsivity are slightly worse than some of state-of-the-art (stand-alone) Ge-on-Si photodetectors [11], this performance is already sufficient for realizing power-efficient receivers with $-15 \mathrm{dBm}$ sensitivity [3]. In addition, it was found that the dark current depends only weakly on device length and temperature $(4.5 \mu \mathrm{A}$ at $-1 \mathrm{~V}$ at $100 \mathrm{C}$, Fig. $4 \mathrm{c})$. As such, we believe that the dark current originates mainly from a parasitic edge effect, which can likely be mitigated in future designs.
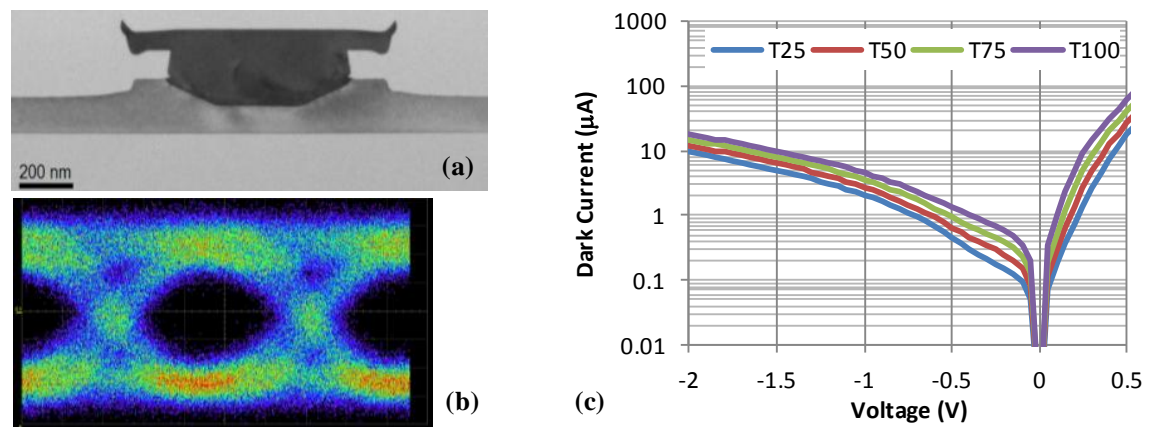

Figure 4: a) TEM image of a Ge waveguide photodectector with laterally overgrown contact areas (prior to contacting module), b) $10 \mathrm{~Gb} / \mathrm{s}$ eye diagram for a $5 \times 2 \mu \mathrm{m}^{2} \mathrm{Ge} \mathrm{PD}$ at $-2 \mathrm{~V}$, c) dark current vs. voltage and temperature for a $10 \times 2 \mu \mathrm{m}^{2} \mathrm{Ge}$ PD.

\section{Conclusion}

The adoption of silicon photonics as a technology for low-power high-bandwidth Optical I/O is contingent upon the co-integration of highly efficient silicon optical devices, including both voltage-efficient silicon modulators and highly responsive Ge photodetectors. Recent results on low-loss, low voltage depletion and accumulation ring modulators have been reviewed, as well as the co-integration with Ge photodetectors.

The authors acknowledge the efforts of imec's 200-mm pline for their contributions to the device fabrication. This work was supported by imec's Core Partner Program.

\section{References}

[1] D. A. B. Miller, "Device Requirements for Optical Interconnects to Silicon Chips," Proc. IEEE 97, 1166 - 1185 (2009)

[2] W. Zhang et al, "Ni/Cu/Sn bumping scheme for fine-pitch micro-bump connections," $61^{\text {st }}$ Electronic Components and Technology Conference (IEEE 2011), pp. $109-113$

[3] X. Zheng, et al, "Ultra-efficient 10Gb/s hybrid integrated silicon photonic transmitter and receiver," Optics Express, Vol. 19, Issue 6, pp. $5172-5186(2011)$

[4] G. Li, X. Zheng, J. Yao, H. Thacker, I. Shubin, Y. Luo, K. Raj, J. E. Cunningham, and A. V. Krishnamoorthy, "25Gb/s 1V-driving CMOS ring modulator with integrated thermal tuning," Optics Express 19(21), 20435-443 (2011).

[5] M. Ziebell, D. Marris-Morini, Gilles Rasigade, P. Crozat, J.-M. Fedeli, P. Grosse, E. Cassan, and L Vivien, "Ten Gbit/s ring resonator silicon modulator based on interdigitated PN junctions," Optics Express 19, 14690-695 (2011).

[6] M. Pantouvaki et al, " Lateral versus Interdigitated Diode Design for $10 \mathrm{~Gb} / \mathrm{s}$ Low-Voltage Low-Loss Silicon Ring Modulators," in IEEE Photonics Society Optical Interconnects Conference (IEEE, 2012), to be presented.

[7] H. Yu et al, "Doping Geometries for 40G Carrier-Depletion-Based Silicon Optical Modulators," in Optical Fiber Communication Conference, OSA Technical Digest (CD) (Optical Society of America, 2012), paper OW4F.4.

[8] J. Van Campenhout et al, "Low-Voltage, Low-Loss, Multi-Gb/s Silicon Micro-Ring Modulator based on a MOS Capacitor," in Optical Fiber Communication Conference, OSA Technical Digest (CD) (Optical Society of America, 2012), paper OM2E.4.

[9] http://www.ofcnfoec.org/osa.ofc/media/Default/PDF/2009/09-Dandrea.pdf

[10] P.Verheyen et al, "Co-integration of Ge detectors and Si modulators in an advanced Si photonics platform," in Photonics Europe, (SPIE, 2012), paper 8431-39, to be presented.

[11] C. T. DeRose et al, "Ultra compact $45 \mathrm{GHz}$ CMOS compatible Germanium waveguide photodiode with low dark current," Optics Express, Vol. 19, Issue 25, pp. 24897-24904 (2011) 